\title{
Inhibitory tagging in inhibition of return: Evidence from flanker interference with multiple distractor features
}

\author{
ANa B. Vivas \\ City Liberal Studies, Thessaloniki, Greece \\ LUIS J. FuENTES \\ Universidad de Murcia, Murcia, Spain \\ Angeles F. Estevez \\ Universidad de Almería, Almería, Spain \\ AND \\ GLYN W. HUMPHREYS \\ University of Birmingham, Birmingham, England
}

\begin{abstract}
Fuentes, Vivas, and Humphreys (1999) proposed a distinction between inhibition of return (IOR) and inhibitory processing taking place at a location subject to IOR. This latter mechanism, inhibitory tagging (IT), would act at a late level of processing related to response selection. In the present study, we examined whether IT was applied only to the target-relevant properties of the stimuli (e.g., to its color) or whether it was applied to other features of the stimulus as well (e.g., to its shape). Both when the task was to respond to the target's color (Experiment 1) as well as when it was to respond to the target's shape (Experiment 2), there was evidence of IT (reversal of the typical flanker effect at the cued location, relative to the uncued location) only to task-relevant features of the target. These findings suggest that IT is a central process of control constrained by task demands and current goals.
\end{abstract}

When attention is summoned by an uninformative exogenous cue that precedes the presentation of a target by more than $300 \mathrm{msec}$, response times (RTs) to stimuli that appear at that location are usually slower than RTs to stimuli that appear at uncued locations. It has been suggested that this effect, termed inhibition of return (IOR), reflects an attentional bias toward novelty (Posner \& Cohen, 1984; Posner, Rafal, Choate, \& Vaughan, 1985). IOR may aid visual search processes in cluttered environments by favoring attentional exploration of new items (Klein, 1988); consistent with this, it has been reported for up to four or five locations cued in sequence (e.g., Danzinger, Kingstone, \& Snyder, 1998; Snyder \& Kingstone, 2000; Takeda \& Yagi, 2000; Tipper, Weaver, \& Watson, 1996).

For performance to be efficiently modulated by this attentional bias for novelty, it would be important to delay not only the orienting of attention to previously attended locations but also the processing of any information appearing at these locations. Fuentes, Vivas, and Humphreys (1999), for example, have proposed that biases against information at previously attended locations arise at a response stage of processing. They suggested that a pro- cess termed inhibitory tagging (IT) was applied to stimuli falling at earlier attended positions, hindering access to associated responses. The application of IT can explain why IOR interacts with processes other than those affecting attentional orienting, including semantic priming, flanker interference (Fuentes et al., 1999), Stroop interference (Vivas \& Fuentes, 2001), and the Simon effect (Fuentes, Vivas, de Labra, Valle-Inclán, \& Alonso, 2002; see also Ivanoff, Klein, \& Lupiáñez, 2002). For example, Fuentes et al. (1999) found that semantic priming and distractor interference effects reverse when the prime and the distractor stimuli, respectively, are presented at the cued location in a IOR procedure; that is, RTs were faster when the prime was unrelated to the target, and/or when the target was flanked by incongruent rather than congruent distractors. In addition, the Stroop effect decreased (Vivas \& Fuentes, 2001; Vivas, Humphreys, \& Fuentes, 2003) and the Simon effect increased (Fuentes et al., 2002) when the target stimuli appeared at a cued location rather than at an uncued location. Although IOR seems to influence all these effects differently (decreasing one, increasing a second, and reversing a third), we

A. B.Vivas, vivas@city.academic.gr 
believe that these results can be explained in terms of IT particularly affecting stimulus-response ( $\mathrm{S}-\mathrm{R}$ ) codes that are either task relevant or task irrelevant, but that are normally derived rapidly. In the flanker task, this temporary disconnection of S-R codes would produce slower RTs to central targets that share the same response with the distractor stimulus, at the cued location (in the compatible flanker condition), relative to those targets that generate the opposite response to the distractor (in the incompatible condition; Fuentes et al., 1999). Disconnecting the compatible response would slow performance, whereas disconnecting the response from incompatible distractors would speed RTs, because any response competition with the target would be lessened. In the same way, if IT at the cued location affected only the task-relevant response, we would expect increased effects of activated but taskirrelevant information - in this case, the irrelevant spatial S-R code - in the Simon spatial-compatibility paradigm, because the relevant response code would be slowed. The Simon effect should therefore increase at the location subject to IOR (Fuentes et al., 2002). Finally, IT could account for the reduction of Stroop interference at the cued location, if it affected S-R codes that are normally derived rapidly, such as the word name in the Stroop task. In this case, the inhibitory link could prevent an incongruent word response from competing with later derived responses to the color name, reducing Stroop interference for targets at cued locations.

Thus, IT may account for a wide range of responserelated effects at the cued location, and it may operate as a flexible central process of control, acting at a response level to modulate processing at the inhibited location. In the present study, we assess a further aspect of IT: namely, whether IT is applied only to task-relevant properties of stimuli or whether it operates on object representations where both task-relevant and task-irrelevant features are combined; in other words, if the task were to select a stimulus on the basis of its shape, would IT apply to other features of the stimulus, such as its color or texture, as well? There is evidence that visual selection may be tuned to task-relevant features and does not necessarily deal with both task-relevant and task-irrelevant features. Thus, negative priming of ignored distractors applies to the features on which the stimuli are selected and not necessarily to others (Tipper, Weaver, \& Houghton, 1994). Similarly, our problems in selecting multiple tokens apply only to the task-relevant dimensions of the stimuli (Baylis, Driver, \& Rafal, 1993). Here we may ask whether the same is true of IT. We had participants discriminate either the color, in Experiment 1, or the shape, in Experiment 2, of targets flanked by distractors that could fall at previously cued or uncued locations. We assessed whether any modulation of flanker interference by the cue was influenced by whether color or shape was relevant in the primary task; for example, was any reversal of flanker interference, when the flanker appeared at a cued location, contingent on the similarity of the task-irrelevant as well as of task-relevant properties of the flanker and the target?

\section{GENERAL METHOD}

\section{Stimuli}

Stimuli were presented on the color monitor (VGA) of an IBM-PC compatible computer, and responses were recorded through the computer keyboard. The software used for creating and running the experiment was Micro Experimental Laboratory (MEL; Schneider, 1988). In the discrimination task, the target stimuli consisted of circles with diameters of $1.2 \mathrm{~cm}$ each and equilateral triangles with sides of $2 \mathrm{~cm}$ each (see Figure 1). The target stimuli always appeared in the central box subtending $4.3^{\circ} \times 2.2^{\circ}$. Distractor stimuli were drawn from the same geometrical shapes as were the targets, and one distractor appeared randomly in one of the peripheral boxes on each trial. The distance between the inner side of the peripheral boxes and the central point was $2.4^{\circ}$. The different congruency conditions were created by orthogonally combining the color and shape dimensions of the target and the distractor (see Figure 1). The target and distractor stimuli could be congruent or incongruent in the task-irrelevant feature (shape in Experiment 1 and color in Experiment 2), and congruent or incongruent in the task-relevant feature (color in Experiment 1 and shape in Experiment 2). In Experiment 2, there was also a distractor stimulus that was neutral with respect to both features (a blue square, the bottom distractor in Figure 1). In each of these conditions, the distractor could appear at either a previously cued or uncued location, under IOR conditions.

In the detection task in Experiment 1, the target stimulus was drawn from the same geometrical figures used as distractors in the discrimination task. In Experiment 2, the target stimulus consisted of an asterisk.

\begin{tabular}{|c|c|c|c|c|}
\hline & & $\begin{array}{c}\text { Color } \\
\text { Congruency }\end{array}$ & & \begin{tabular}{|l}
$\boldsymbol{G}$ Red \\
Green \\
Blue
\end{tabular} \\
\hline $\begin{array}{l}\text { Shape } \\
\text { Congruency }\end{array}$ & $\begin{array}{l}\text { Congruent } \\
\text { Distractor }\end{array}$ & $\begin{array}{l}\text { Incongruent } \\
\text { Distractor }\end{array}$ & $\begin{array}{c}\text { Neutral } \\
\text { Distractor }\end{array}$ & \\
\hline Congruent & & & & \\
\hline Incongruent & & & & \\
\hline Neutral & & & & \\
\hline
\end{tabular}

Figure 1. Stimuli employed in the discrimination task. 


\section{Procedure}

Participants were seated approximately $60 \mathrm{~cm}$ from the computer's screen. Figure 2 shows the sequence of stimuli and the duration of exposure. On each trial, a fixation point (a small cross) appeared in the middle of the screen for $500 \mathrm{msec}$ and was then replaced by three white boxes presented for $1,000 \mathrm{msec}$. One of the peripheral boxes subsequently thickened for $300 \mathrm{msec}$ (the peripheral cue). This was followed by a temporal interval of $200 \mathrm{msec}$ with the three boxes, and then the central box thickened for $300 \mathrm{msec}$ (the central cue). After a further interval of $200 \mathrm{msec}$, the target display was presented for 2,000 msec, or until participants responded.

In the detection task, the target display consisted of one stimulus presented inside one of the lateral boxes. Each participant was required to detect the target by pressing the space bar on the computer's keyboard, using the nondominant hand. In the discrimination task, the critical display consisted of the target stimulus presented in the central box, flanked by one distractor stimulus presented inside one of the lateral boxes. Each participant was required to respond to the central stimulus by pressing one of two little patches attached to two keys of the keyboard (for half of the participants, the red circular and green triangular patches were attached to the "J" and " $\mathrm{K}$ " keys, respectively, and the opposite was true for the rest of the participants) and to press the keys with the first and middle fingers of the dominant hand. The detection and discrimination tasks were mixed randomly within blocks. Participants knew which task to perform on the basis of whether the target display contained one or two stimuli (one stimulus $=$ detection; two stimuli $=$ discrimination of the central target).

\section{EXPERIMENT 1 Color Discrimination Task}

In order to study the effect of IOR on both task-relevant and task-irrelevant S-R codes, in the present experiment we used stimuli that could vary in two features, color (green or red) and shape (a triangle or a circle). Consequently, in Experiment 1, the distractor stimulus could be congruent or incongruent to the target in the task-relevant feature (color), and congruent or incongruent in the task-irrelevant feature (shape). Participants performed one of two intermingled tasks. In the detection task, performed on $75 \%$ of the trials, they responded to the presence of a stimulus (a circle or a triangle colored red or green) at one of the two peripheral locations. This enabled us to generate a direct measure of IOR in the study. In the discrimination task (on $25 \%$ of the trials), participants responded to the color of a central stimulus (a circle or a triangle colored red or green) that was presented simultaneously with a distractor stimulus at one of the two peripheral locations. This enabled us to measure the effects of IT on distractor processing.

If IT affected only the task-relevant feature, then when the distractor appears at the cued location we should find a reversal of the standard flanker interference, on the basis of the color relations between the stimuli (RTs being slower when the distractor has a congruent rather than an incongruent color). Whether the distractor shape is congruent or incongruent with respect to the target should be irrelevant to performance.

\section{Method}

Participants. Twenty undergraduate students from the University of Almería participated in the experiment. They received course credits for their participation, and all had normal or corrected-tonormal vision.

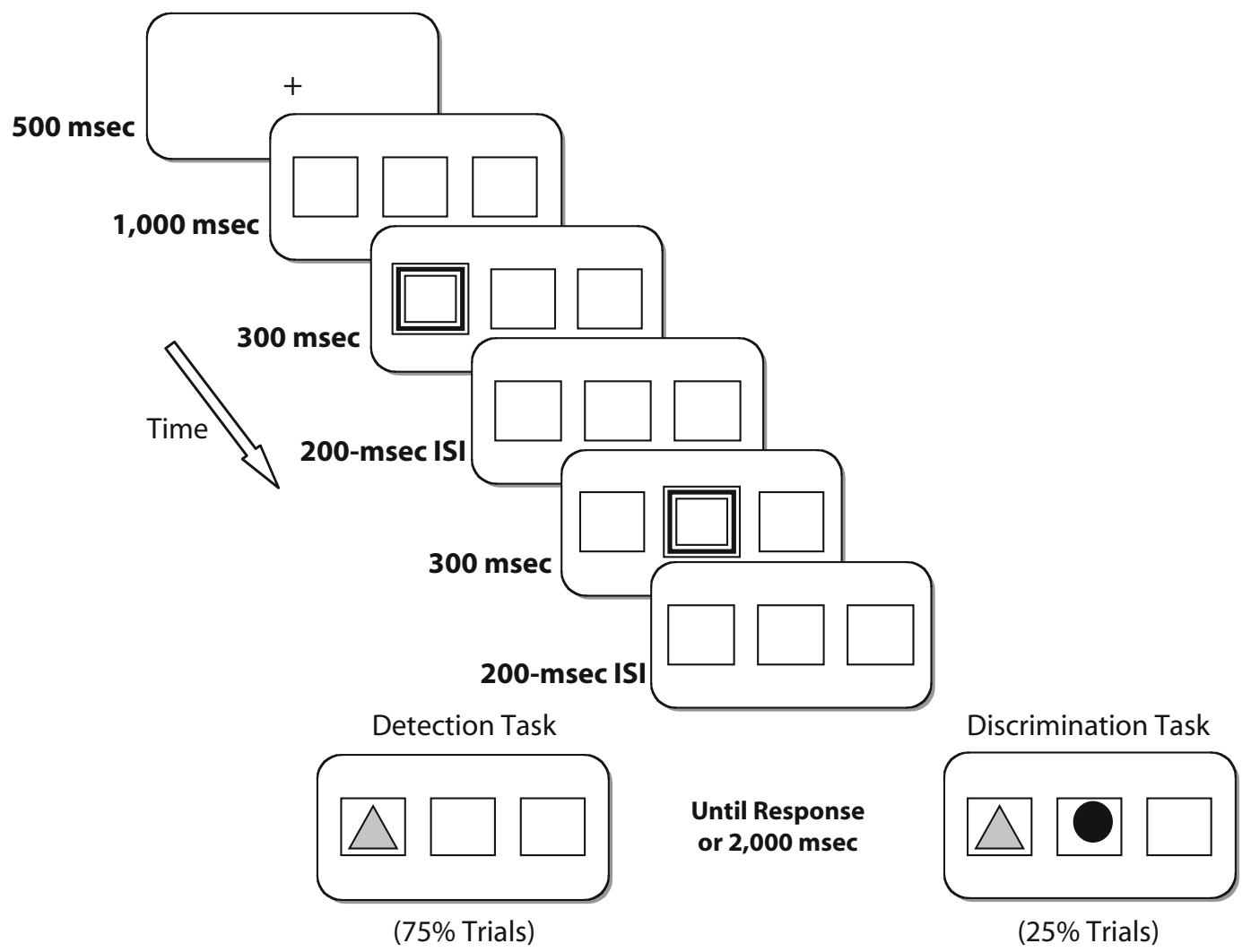

Figure 2. Sequence of events and duration of stimuli in Experiments 1 and 2. 
Design. There was one experimental block of 256 trials preceded by one practice block. On $75 \%$ of the trials of the experimental block (192 trials) there was a single stimulus in one of the peripheral boxes. On half of these trials (96 trials) the stimulus appeared at a cued location and on the remaining trials at an uncued location. On $25 \%$ of the trials (64 trials), the target stimulus was presented in the central box flanked by a distractor stimulus in one of the peripheral boxes. The distractor appeared at the cued location for half of these trials (32 trials), and at the uncued location for the other half. On half of these trials ( 16 trials), the distractor and the target were congruent in the task-irrelevant feature (shape) and incongruent on the remaining 16 trials. Finally, within each set of 16 trials, there were 8 trials for each task-relevant (color) congruency condition.

\section{Results and Discussion}

The error analyses did not show any significant effect either for the detection or for the discrimination tasks (all $F \mathrm{~s}<1$ ); errors are not further discussed (see Table 1 for the data).

In the detection task, correct RTs were submitted to a repeated measures ANOVA with location (cued and uncued) as the within-subjects factor. RTs were longer when the target appeared at the cued location than when it appeared at the uncued location (479 vs. $457 \mathrm{msec}$, respectively) $[F(1,19)=15.48, p<.005]$. This confirms that there was an IOR effect.

In the discrimination task, correct RTs were submitted to a $2 \times 2 \times 2$ repeated measures ANOVA with distractor stimulus location (cued and uncued), task-relevant congruency (color congruent and color incongruent) and taskirrelevant congruency (shape congruent and shape incongruent) as within-subjects factors (see Table 1). The results showed a main effect of distractor location $[F(1,19)=$ $17.74, p<.001]$. RTs were significantly shorter when the distractor appeared at the cued location $(649 \mathrm{msec})$ compared with the uncued location (677 $\mathrm{msec})$.

The two-way interaction between location and taskrelevant (color) congruency was also significant $[F(1,19)=$ $12.1, p<.05]$. When the distractor fell at the uncued location, we observed the standard flanker effect; that is, RTs were shorter when the distractor and the target were congruent in color $(665 \mathrm{msec})$ than when they were incon-

Table 1

Mean RTs and Percentage of Errors As a Function of the Location of the Distractor Stimulus, Task-Irrelevant Congruency (TIC), and Task-Relevant Congruency (TRC) to the Target in Experiments 1 and 2

\begin{tabular}{|c|c|c|c|c|c|c|}
\hline \multirow[b]{3}{*}{ Location } & \multirow[b]{3}{*}{ TIC } & \multicolumn{4}{|c|}{ TRC } & \multirow[b]{3}{*}{ FCE } \\
\hline & & \multicolumn{2}{|c|}{ Congruent } & \multicolumn{2}{|c|}{$\underline{\text { Incongruent }}$} & \\
\hline & & $M$ & $\% \mathrm{E}$ & $M$ & $\% \mathrm{E}$ & \\
\hline \multicolumn{7}{|c|}{ Experiment 1} \\
\hline \multirow[t]{2}{*}{ Cued } & $\mathrm{C}$ & 664 & 1.5 & 623 & 3.3 & $-41^{*}$ \\
\hline & I & 655 & 4.0 & 655 & 4.0 & 0 \\
\hline \multirow[t]{2}{*}{ Uncued } & $\mathrm{C}$ & 653 & 2.7 & 691 & 4.4 & $38^{*}$ \\
\hline & I & 677 & 1.6 & 687 & 3.2 & 10 \\
\hline \multicolumn{7}{|c|}{ Experiment 2} \\
\hline \multirow[t]{2}{*}{ Cued } & $\mathrm{C}$ & 716 & 2.3 & 682 & 1.5 & $-34^{*}$ \\
\hline & I & 685 & 4.5 & 675 & 3.0 & 10 \\
\hline \multirow[t]{2}{*}{ Uncued } & $\mathrm{C}$ & 700 & 4.5 & 746 & 2.3 & $46^{*}$ \\
\hline & I & 713 & 1.5 & 708 & 1.7 & -5 \\
\hline
\end{tabular}

Note-FCE, flanker compatibility effect; C, congruent; I, incongruent. $\quad{ }^{*} p<.05$. gruent (689 msec). However, this effect reversed when the distractor fell at the cued location, where RTs were longer for the congruent condition $(659 \mathrm{msec})$ than for the incongruent condition $(639 \mathrm{msec})$. This pattern of results replicates Fuentes et al.'s (1999) findings of a reversal on the standard congruency effect in the flanker task when the flanker falls at a previously cued location. However, these effects were modulated by a significant location $\times$ taskrelevant congruency $\times$ task-irrelevant congruency interaction $[F(1,19)=17.06, p<.05]$. When the task-irrelevant feature (shape) was congruent with the target, least significant difference (LSD) post hoc comparisons showed a significant flanker effect (38 msec of effect) at the uncued location $(p<.05)$, but this effect significantly reversed $(-41 \mathrm{msec}$ of effect) when the flanker stimulus appeared at the cued location $(p<.05)$. When the task-irrelevant feature was incompatible with the target, LSD post hoc comparisons showed no significant differences between congruent and incongruent distractors (effect sizes of 0 and $10 \mathrm{msec}$ for the cued and the uncued location, respectively) for any of the location conditions ( $p s>.05)$.

These results fit the general pattern expected if IT took place at the cued but not at the uncued location. When the distractor fell at the cued location, IT of its properties would slow RTs to targets sharing the same task-relevant properties (the color-congruent condition) as the colorincongruent condition. In contrast, when the distractor fell at the uncued location, responses based on its features were not inhibited, so that a color-congruent distractor facilitated responses to the target and a color-incongruent distractor disrupted them. The new result here is that the flanker effect, and consequently the IT effects, emerged as a function of whether the target also shared its shape (task-irrelevant feature) with the distractor. However, mismatch in the task-irrelevant feature seems to have also eliminated the response compatibility effect at the uncued location (a nonsignificant effect size of $10 \mathrm{msec}$ ). A possible explanation of this unexpected result could be that selective attention to the location of the distractor led to the integration of both features into a single representation, although only one feature was relevant for the task. If target-distractor congruency is then defined on the basis of the color-shape combination, only the condition in which target and distractor were identical would be congruent, whereas all other conditions would be incongruent. In agreement with this hypothesis, when we averaged the nonidentical conditions we found that the identical condition was significantly shorter (653 msec) than the incongruent-average condition $(685 \mathrm{msec})$ at the uncued location $[t(19)=3.55, p<.05]$. Our results thus suggest that task-irrelevant features of the stimuli modulate flanker compatibility effects.

\section{EXPERIMENT 2 Shape Discrimination Task}

Experiment 2 investigated whether the reversal of the color flanker compatibility effect in Experiment 1 could be modulated by task relevancy. In order to test this, we employed the same procedure as in Experiment 1, but now 
participants had to respond to the shape of the central target. We assessed whether the IT effect was contingent only on the task-relevant features (e.g., the shape of the distractors), or whether irrelevant features also influenced performance.

In addition, we introduced two changes in the procedure to the first study: (1) in the detection task, the target stimulus was an asterisk; and (2) a neutral flanker stimulus (see Figure 1) was included. The neutral flanker stimulus would allow us to determine whether the reversal of the flanker effect was due to changes in the incongruent condition, in the congruent condition, or in both. The change to using an asterisk target in the detection task was to prevent participants' responding to irrelevant flankers on $75 \%$ of the trials.

\section{Method}

Participants. Twenty undergraduate students from the University of Almería participated in the experiment. They received course credits for their participation, and all of them had normal or corrected-to-normal vision.

Design. On $75 \%$ of the trials (240 trials), an asterisk appeared, cuing a detection response. This asterisk fell at the previously cued location on half of the trials (120 trials), and at the uncued location for the other half. On $25 \%$ of the trials ( 80 trials), the target stimulus was presented in the central box flanked by a distractor stimulus in one of the peripheral boxes. This cued a discrimination response to the central item. The distractor appeared at the cued location for half of these trials (40 trials), and at the uncued for the other half. Finally, there were 8 trials for each congruency condition, congruent on both the task-relevant and the task-irrelevant feature (CC), incongruent on the task-relevant feature and congruent on the taskirrelevant feature (IC), congruent on the task-relevant feature and incongruent on the task-irrelevant feature (CI), incongruent on both the task-relevant and the task-irrelevant feature (II) and neutral (N). Each congruency condition was, therefore, represented on one fifth of the total discrimination trials.

\section{Results and Discussion}

Four participants were eliminated from the data analyses because of a high percentage of errors (more than 30\% of trials). The error analyses did not reveal any significant effect for either task (all $p \mathrm{~s}>.05$ ) (see Table 1 for the data).

In the detection task, RTs were submitted to a repeated measures ANOVA with location (cued and uncued) as the within-subjects factor. The results showed $19 \mathrm{msec}$ of IOR effect, a main effect of location $[F(1,15)=20.94$, $p<.001]$.

In the discrimination task, a $2 \times 2 \times 2$ repeated measures ANOVA was performed on correct RTs, with location of the distractor stimulus (cued and uncued), task-irrelevant congruency (color congruent and color incongruent) and task-relevant congruency (shape congruent and shape incongruent) as within-subjects factors (see Table 1). The results showed significant main effects of location and task-irrelevant congruency $[F(1,15)=9.91$, $p<.05$, and $F(1,15)=5.56, p<.05$, respectively]. That is, RTs were slower when the distractor fell at the uncued location $(716 \mathrm{msec})$ than when it fell at the cued location (689 msec). Also, RTs were higher for the color-congruent condition $(710 \mathrm{msec})$ than for the color-incongruent condition (695 msec).
Most important, the location $\times$ task-relevant congruency interaction was significant $[F(1,15)=7.8, p<.05]$. The standard response compatibility effect, for the relevant target dimension, was found when the distractor appeared in the uncued location $(-21 \mathrm{msec})$. However, this reversed when the distractor fell at the cued location (22-msec effect). This pattern of results replicated the findings of Fuentes et al. (1999) and those found in Experiment 1. As in the previous experiment, the location $\times$ task-relevant $\times$ task-irrelevant interaction was also significant $[F(1,15)=5.88, p<.05]$. LSD post hoc comparisons showed a significant response compatibility effect (a 46-msec effect) at the uncued location when the task-irrelevant feature was compatible with the target $(p<.05)$; whereas this effect significantly reversed $(-34 \mathrm{msec}$ of effect) when the distractors appeared at the cued location and the task-irrelevant feature was compatible with the target $(p<.05)$. However, when the taskirrelevant feature was incompatible with the target, there were no significant differences between compatible and incompatible distractors for either the cued $(-5 \mathrm{msec}$ of effect) or uncued $(10 \mathrm{msec})$ location $(p \mathrm{~s}>.05)$.

Finally, in order to analyze the neutral condition, RT data were submitted to a $2 \times 5$ repeated measures ANOVA with location (cued and uncued) and congruency (CC, IC, CI, II, and N) as within-subjects factors. The analyses showed a main significant effect of location $[F(15,60)=$ $7.04, p<.05]$ and a significant location $\times$ congruency interaction $[F(15,60)=4.21, p<.05]$. Planned $t$ test analyses at the cued location showed that the neutral baseline (732 msec) significantly differed from the IC (682 msec), CI $(685 \mathrm{msec})$, and II $(675 \mathrm{msec})$ conditions $[t(16)=$ $3.71, p<.05 ; t(16)=2.77, p<.05 ;$ and $t(16)=3.6, p<$ .05 , respectively]. Planned $t$ test analyses at the uncued location showed that the neutral baseline $(719 \mathrm{msec})$ did not significantly differ from any of the other congruency conditions $(p \mathrm{~s}>.05)$.

We found IOR in the detection task when a high proportion of peripheral targets were employed and a unique target (an asterisk) was used for detection. Most important, as in Experiment 1, we found that an IT process, which acts at the location subject to IOR, modulated the impact of the distractor on the responses to the target. In other words, the typical flanker compatibility effect found at the uncued location reversed when the distractor stimulus was presented at the cued location. Furthermore, this interaction was observed only when the task-irrelevant feature of the target matched the distractor stimuli. Finally, as in Experiment 1, the incongruent task-irrelevant condition also failed to produce significant flanker compatibility effects at the uncued location (an effect of only $-5 \mathrm{msec}$ was observed). Thus, the results from Experiment 2 mirrored those from Experiment 1.

The present results suggest that both congruency facilitation and its reversal operate more strongly when the flanker and the target are identical. As suggested above, selective attention to the location of the distractor may have led to the integration of both relevant and irrelevant features into a single representation. On the basis of a combined color-shape criterion, then, only the identical 
condition would be congruent, whereas the other combinations of color and shape would be to some extent incongruent. In agreement with this hypothesis, and similar to Experiment 1, the identical condition $(700 \mathrm{msec})$ was significantly shorter that the averaged incongruent condition (722 msec) at the uncued location $[t(19)=1.28, p<$ $.05]$.

One difference between Experiments 1 and 2 is that in Experiment 2 we included a neutral flanker stimulus to provide an estimate of whether the interaction between IOR and flanker compatibility was due to changes in the congruent condition, or to changes in the incongruent condition, or to both. Unfortunately, results showed that the neutral stimulus was not particularly useful as a baseline, since RTs in this condition were relatively high for both location conditions (732 msec for the cued condition and $719 \mathrm{msec}$ for the uncued condition). Given that neutral trials (blue squares) occurred on $20 \%$ of the trials on the discrimination task, whereas on $80 \%$ of the trials a red or green stimulus appeared, neutral trials may have violated general expectations in the study, and may have consequently led to slow RTs being made to the target. ${ }^{1}$

Finally, the results from this second experiment also allow us to conclude that the interaction between flanker and IOR effect found in Experiment 1 cannot be explained as an artifact of the distractor stimulus being the target on $75 \%$ of the trials (in the detection task), because we obtained the same results when we presented an asterisk as the target stimulus in the detection task (Experiment 2).

\section{GENERAL DISCUSSION}

The results from the present study show that (1) IT modulates stimulus processing at the cued location, regardless of whether the task is to select a stimulus on the basis of its color (Experiment 1) or its shape (Experiment 2), and (2) there was evidence of IT (reversal of the typical flanker effect at the cued location relative to the uncued location) only to task-relevant features of the target. Although the interaction between IOR and task-relevant congruency was also modulated by task-irrelevant congruency, the lack of flanker compatibility effects at the uncued location for this condition does not allow us to draw conclusions about IT being applied to task-irrelevant response codes.

The present results are important for different reasons. First, although a considerable number of experiments have investigated the mechanisms underlying IOR, few studies have examined how stimulus processing is affected in locations subject to IOR. The present study therefore adds to a growing body of evidence of impaired access to responses associated with stimuli at the cued location. Recently, however, Ro, Machado, Kanwisher, and Rafal (2002) used a similar procedure to the one employed here and found that the magnitude of the flanker effect was not influenced by the cuing manipulation. Procedural differences relating to the cue-target SOA (Ro et al., 2002, used a shorter interval of $750 \mathrm{msec}$ ) and the presentation conditions (the target display remained on the screen for only $17 \mathrm{msec}$ in their study) may account for this discrepancy. Further studies in which boundary conditions of IT were examined may explain why different results arose in these cases. Second, and more importantly, the findings from the present study suggest that IT is a flexible central process of control constrained by task demands and current goals. Thus, IT seems to affect response codes activated by the target features relevant to the current task goals (Fuentes et al., 2002; Experiments 1 and 2 in the present study) or those that are prepotent, because normally they are derived more rapidly, as in the case of naming the word in the Stroop task (Vivas \& Fuentes, 2001). This agrees with other attentional explanations of effects, such as negative priming and repetition blindness (Baylis et al., 1993; Tipper et al., 1994), which propose selection processes that operate on task-relevant features.

Furthermore, studies with neurological patients and healthy older adults suggest that IOR and IT can be differentially affected by brain damage and aging (Fuentes, Boucart, Vivas, Alvarez, \& Zimmerman, 2000; Langley, Vivas, Fuentes, \& Bagne, 2005; Vivas et al., 2003). Vivas and Fuentes (2001) investigated IT with a group of schizophrenic patients (Fuentes et al., 2000), whereas other studies have assessed effects of damage to the posterior parietal lobe (Vivas et al., 2003) and of aging (Langley et al., 2005). In the study with schizophrenic patients, there were normal IOR and Stroop effects, compared with the control group, but as opposed to the control group, the patients did not demonstrate the modulation of Stroop interference at the cued location. We concluded that IT is impaired in schizophrenia (Fuentes et al., 2000). On the other hand, the patients with lesions to the posterior parietal cortex showed evidence of IT (decreased Stroop interference at the cued location relative to the uncued location) but only for the visual field that also elicited IOR (the contralesional visual field). These results suggest that IOR is needed to produce the IT effect, but, following the findings from schizophrenia, IOR is not sufficient (Vivas et al., 2003). Finally, the data from Langley et al. suggest that IOR is more resistant to aging than is IT. Thus, whereas younger adults showed evidence of both IOR and IT, the group of older adults demonstrated equivalent IOR effects, but failed to show the interaction between IOR and Stroop interference. This pattern of results is in agreement with other studies, which indicate that age does not affect all forms of cognitive inhibition in a uniform fashion and that stimulus-based inhibitory processes (e.g., IT) are more affected by aging than are location-based inhibitory processes (e.g., IOR; Langley et al., 2005). Taking all these findings together, we suggest (Langley et al., 2005; Vivas et al., 2003) that the implementation of IT may be related to the functioning of anterior cortical areas that would receive a signal from more posterior areas involved in the implementation of spatial, location-based, inhibitory bias (IOR). Regardless of this proposal, though, the results indicate that, while the two inhibitory effects can be dissociated, they normally cooperate to effectively bias organisms toward novelty.

\section{AUTHOR NOTE}

This work was supported by Grants BSO2003-04594 (to L.J.F.) and BSO2003-00930 (to A.F.E.) from the Ministerio de Ciencia y Tec- 
nología, and by a Medical Research Council grant to G.W.H. We thank Tram Neill, Bruce Milliken, Jay Pratt, and an anonymous reviewer for their comments on an earlier version of this manuscript. Correspondence concerning this article should be addressed to A. B. Vivas, Department of Psychology, City Liberal Studies (Affiliated Institution of the University of Sheffield), 13 Tsmiski St., 54624 Thessaloniki, Greece (e-mail: vivas@city.academic.gr) or to L. J. Fuentes, Dpto. Psicología Básica y Metodología, Universidad de Murcia, 30100 Murcia, Spain (e-mail: lfuentes@um.es).

Note-Accepted by David A. Balota's editorial team.

\section{REFERENCES}

Baylis, G. C., Driver, J., \& Rafal, R. D. (1993). Visual extinction and stimulus repetition. Journal of Cognitive Neuroscience, 5, 453-466.

Danzinger, S., Kingstone, A., \& Snyder, J. J. (1998). Inhibition of return to successively stimulated locations in a sequential visual search paradigm. Journal of Experimental Psychology: Human Perception \& Performance, 24, 1467-1475.

Fuentes, L. J., Boucart, M., Vivas, A. B., Alvarez, R., \& ZimmerMAN, M. A. (2000). Inhibitory tagging in inhibition of return is affected in schizophrenia: Evidence from the Stroop task. Neuropsychology, 14, 134-140.

Fuentes, L. J., Vivas, A. B., De Labra, C., Valle-Inclán, F., \& Alonso, D. (2002, April). Visuospatial attention and stimulusresponse compatibility. Paper presented at the 4th Spanish Experimental Society (SEPEX) meeting, Oviedo, Spain.

Fuentes, L. J., Vivas, A. B., \& Humphreys, G. W. (1999). Inhibitory tagging of stimulus properties in inhibition of return: Effects on semantic priming and flanker interference. Quarterly Journal of Experimental Psychology, 52A, 149-164.

IVANOFF, J., KLEIN, R. M., \& LuPIÁÑEZ, J. (2002). Inhibition of return interacts with the Simon effect: An omnibus analysis and its implications. Perception \& Psychophysics, 64, 318-327.

KLEIN, R. M. (1988). Inhibitory tagging system facilitates visual search. Nature, 334, 430-431.

Langley, L. K., Vivas, A. B., Fuentes, L. J., \& Bagne, A. G. (2005) Differential age effects on attention-based inhibition: Inhibitory tagging and inhibition of return. Psychology \& Aging, 20, 356-360.
Posner, M. I., \& Cohen, Y. A. (1984). Components of visual orienting. In H. Bouma \& D. G. Bouwhuis (Eds.), Attention and performance X: Control of language processes (pp. 531-556). Hillsdale, NJ: Erlbaum.

Posner, M. I., Rafal, R. D., Choate, L. S., \& Vaughan, J. (1985). Inhibition of return: Neural basis and function. Cognitive Neuropsychology, 2, 211-228.

Ro, T., Machado, L., Kanwisher, N., \& Rafal, R. D. (2002). Covert orienting to the locations of targets and distractors: Effects on response channel activation in a flanker task. Quarterly Journal of Experimental Psychology, 55A, 917-936.

SchneIder, W. (1988). Micro Experimental Laboratory: An integrated system for IBM PC compatibles. Behavior Research Methods, Instruments, \& Computers, 20, 206-217.

Snyder, J. J., \& Kingstone, A. (2000). Inhibition of return and visual search: How many separate loci are inhibited? Perception \& Psychophysics, 62, 452-458.

TAKEDA, Y., \& YAGI, A. (2000). Inhibitory tagging in visual search can be found if search stimuli remain visible. Perception \& Psychophysics, 62, 927-934.

Tipper, S. P., Weaver, B., \& Houghton, G. (1994). Behavioural goals determine inhibitory mechanisms of selective attention. Quarterly Journal of Experimental Psychology, 47A, 809-840.

Tipper, S. P., Weaver, B., \& Watson, F. (1996). Inhibition of return to successively cued spatial locations: A commentary on Pratt and Abrams (1995). Journal of Experimental Psychology: Human Perception \& Performance, 22, 1290-1293.

Vivas, A. B., \& Fuentes, L. J. (2001). Stroop interference is affected in inhibition of return. Psychonomic Bulletin \& Review, 8, 315-323.

Vivas, A. B., Humphreys, G. W., \& Fuentes, L. J. (2003). Inhibitory processing following parietal damage. Neuropsychologia, 41, 1531-1540.

\section{NOTE}

1. We thank Bruce Milliken for suggesting this explanation of the data.

(Manuscript received October 16, 2002; revision accepted for publication May 31, 2006.) 